\title{
A Projection System for Real World Three-Dimensional Objects Using Spatial Light Modulators
}

\author{
Unnikrishnan Gopinathan, David S. Monaghan, Bryan M. Hennelly, Conor P. Mc Elhinney, Damien P. Kelly, \\ John B. McDonald, Thomas J. Naughton, and John T. Sheridan
}

\begin{abstract}
We discuss a projection system for real world threedimensional objects using spatial light modulators (SLM). An algorithm to encode the digital holograms of real world objects on to an SLM is presented. We present results from experiments to project holograms of real world holograms using a nematic liquid crystal SLM. We discuss the case when the pixel sizes of the charge-coupled device (CCD) and SLM used for recording the hologram and projection are different.
\end{abstract}

Index Terms-Holography, liquid crystal displays, spatial light modulators, three-dimensional (3D) displays.

\section{INTRODUCTION}

D IGITAL holographic techniques to capture, process and display three-dimensional (3D) information broadly falls into three categories: 1) recording of optical wavefront as digital hologram and numerically reconstructing the field [1]-[5]; 2) digital synthesis of holograms and optical reconstruction using liquid crystal devices (LCDs) [6]-[9]; and 3) recording of optical wavefronts as digital holograms and optical reconstruction using LCDs [10]-[12]. The techniques in the third category are of most interest for 3D TV applications as they can capture, process, and display real world 3D information. Of the two main digital holographic techniques to record 3D information, in-line holography has lower sampling requirements as compared to off-axis holography but needs more than one data frame to extract wavefront information [1]. The optical reconstruction may be performed by displaying the optical wavefront using a spatial light modulator (SLM) and the fidelity depends to a large extent on how accurately this can be done.

Manuscript received June 7, 2007; revised September 17, 2007 and October 22, 2007. This work was supported by Enterprise Ireland and Science Foundation Ireland through the Research Innovation and Proof of Concept Funds, and the Basic Research and Research Frontiers Programmes, and also by The Irish Research Council for Science, Engineering and Technology. The work of David S.Monaghan was supported by SPIE The International Society for Optical Engineering for an SPIE Educational Scholarship.

U. Gopinathan, D. S. Monaghan, and J. T. Sheridan are with the School of Electrical, Electronic \& Mechanical Engineering, College of Engineering, Mathematical and Physical Sciences, University College Dublin, Belfield, Dublin 4, Ireland (e-mail: john.sheridan@ucd.ie).

B. M. Hennelly, C. P. Mc Elhinney, and J. B. McDonald are with the Department of Computer Science, National University of Ireland, Maynooth, County Kildare, Ireland.

D. P. Kelly is with the Institut für Photonik \& Zentrum für Mikro- und Nanostrukturen, Technische Universität Wien, A-1040 Wien, Austria.

T. J. Naughton is with Department of Computer Science, National University of Ireland, Maynooth, County Kildare, Ireland., and also with the University of Oulu, RF Media Laboratory, Oulu Southern Institute, 84100 Ylivieska, Finland.

Digital Object Identifier 10.1109/JDT.2007.913943

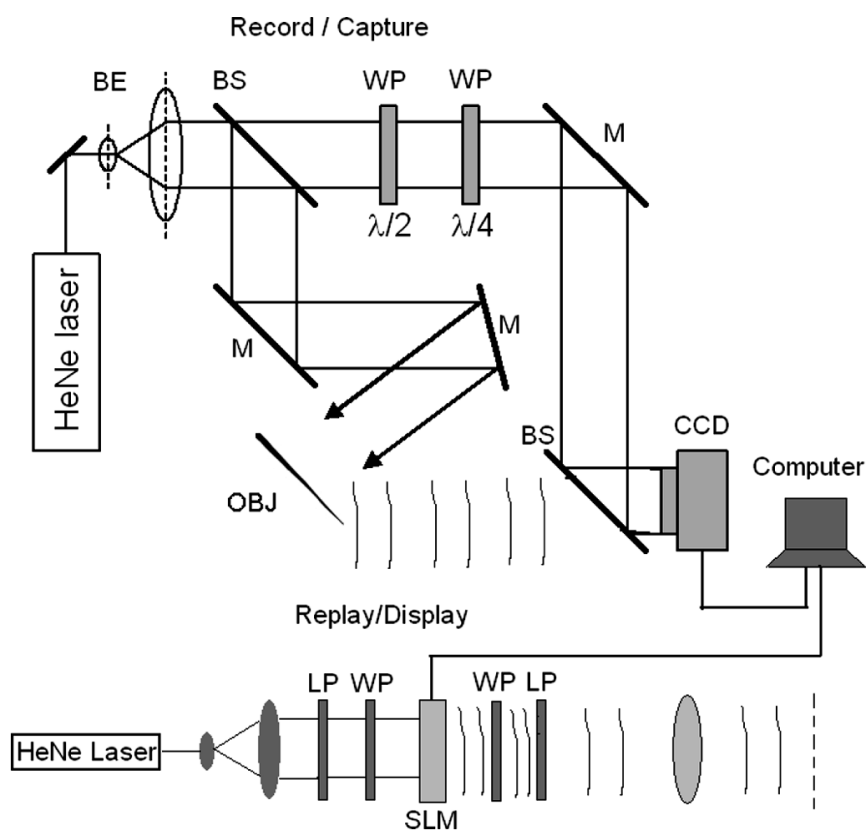

Fig. 1. Schematic of the projection system: BE, beam expander; BS, beam splitter; M, mirror; WP, wave plate; LP, linear polarizer; OBJ, object.

Real SLMs can represent only a limited set of complex values. A number of techniques have been proposed to map a fully complex valued signal onto an SLM that is not fully complex (that can represent only a limited set of complex values) [13]-[17]. A technique to extend the complex modulation range of an SLM pixel with limited modulation states using a combination of linear polarizers and waveplates at the input and output side of the SLM was proposed recently [18].

In this paper, we extend the method proposed in [18] to encode a complex image onto an SLM with limited modulation states. We use this method to demonstrate a projection system for real world objects. We also discuss the factors which affect the optical reconstruction. We present some experimental results obtained using the projection system. We also discuss the factors that affect the optical reconstruction. We present some experimental results obtained using the projection system.

\section{Projection of Real World 3D Objects}

The optical system used for projection of real world 3D objects is shown in Fig. 1. The system consists of a holographic setup to record the digital hologram of the 3D object and a projection system using a SLM. A Mach-Zehnder interferometer is 
used to record the digital holograms. The object to be recorded is placed in one arm (object arm). The other arm (reference arm) contains either a half-wave and or a quarter-wave plate to introduce phase shifts corresponding to $0, \pi / 2, \pi$, and $3 \pi / 2$ rads. The phase of complex wavefront $\mathrm{H}(\cdot)$ at the CCD plane can be calculated from the corresponding four intensity images [2]. The estimated wavefront in the CCD plane must then be encoded to values which an SLM can display. The configuration of polarization elements (linear polarizer and waveplate) at the input and output of the SLM in addition to the voltage, which drives an SLM pixel, determines the complex modulation $\Gamma$ achieved by the SLM given by

$$
\Gamma=\mathrm{P}^{+}\left(\chi_{\text {out }},-\varphi_{\text {out }}\right) \mathrm{M}(\mathrm{V}) \mathrm{P}\left(\chi_{\text {in }}, \varphi_{\text {in }}\right)
$$

where

$$
\mathrm{P}(\chi, \varphi)=\left[\begin{array}{c}
\cos \chi \\
\exp (\mathrm{i} \phi) \sin \chi
\end{array}\right]
$$

is the Jones vector representation of a polarization state with azimuth $\chi$ and angle $\varphi . \chi_{\text {in }}$ and $\chi_{\text {out }}$ are the orientation of the linear polarizers at the input and output side of the SLM. $\varphi_{\text {in }}$ and $\varphi_{\text {out }}$ are the retardance of the waveplates at the input and output side of the SLM. All the angles are specified with respect to the reference laboratory axis chosen to be the axis aligned along the molecular axis at the input face of the SLM. M(V is defined to be the Jones matrix of an SLM [18] pixel written as a function of the applied voltage ' $V$ ' given by

$$
\mathrm{M}(\mathrm{V})=\exp (\mathrm{i} \beta) \mathrm{R}(\alpha)\left[\begin{array}{cc}
\cos \rho \mathrm{e}^{-\mathrm{i} \eta} & -\sin \rho \\
-\sin \rho & \cos \rho \mathrm{e}^{\mathrm{i} \eta}
\end{array}\right]
$$

where $\alpha$ is the twist angle and $\beta$ is the phase shift due to the birefringence of LCD molecules. The parameters $\rho$ and $\eta$ and $\beta$ are functions of applied voltage to the LCD pixel.

The discrete form of (1) may be rewritten as:

$$
\Gamma_{\mathrm{i}}^{(\ell, \mathrm{m})}=\mathrm{P}_{\mathrm{m}}^{+} \mathrm{M}_{\mathrm{i}} \mathrm{P}_{\ell} .
$$

The set of points $\Gamma_{\mathrm{i}}^{(\ell, \mathrm{m})}$ corresponding to a given pair of polarization states $\mathrm{P}_{\ell}$ and $\mathrm{P}_{\mathrm{m}}$ at the input and output of the SLM constitute an operating curve. Each point in this set corresponds to a distinct value of Jones matrix $\mathrm{M}_{\mathrm{i}}$. The desired operating curve determines the choice of configuration of polarization elements at the input and output of the SLM. The choice of the optimum operating curve depends on two factors: 1) the fully complex signal to be mapped and 2) the performance metric of interest. To select the optimum operating curve first, the complex valued image $\mathrm{H}$ is mapped to all possible operating curves. This is done by encoding each value of the $\mathrm{H}$ to an SLM modulation state that is the closest in Euclidean sense (chosen to minimize the Euclidean distance). Each so obtained image $\mathrm{H}^{(\ell, \mathrm{m})}$ is used to evaluate a performance metric. The image that optimizes the performance metric is chosen as the encoded image and the corresponding operating curve determines the configurations of the polarization elements at the input and output of the SLM.

The two performance criteria of interest in the reconstruction of holograms are: 1) the error between the reconstructions of the original and encoded holograms and 2) the diffraction efficiency of the reconstructed holograms. The two performance metrics of interest in this case are defined following [20], [21] as

1) the Amplitude Error $(\mathrm{AE}), \mathrm{E}^{(\ell, \mathrm{m})}$, between the reconstruction of $\mathrm{H}^{(\ell, \mathrm{m})}$ and the reconstruction of $\mathrm{H}$ is quantified using the Amplitude Error given by

$$
\frac{\mathrm{E}^{(\ell, \mathrm{m})}=\sum_{\mathrm{x}, \mathrm{y} \in \mathrm{ROI}}\left[|\mathrm{G}(\mathrm{x}, \mathrm{y})|-\mathrm{k} *\left|\mathrm{G}^{(\ell, \mathrm{m})}(\mathrm{x}, \mathrm{y})\right|\right]^{2}}{\sum_{(\mathrm{x}, \mathrm{y}) \in \mathrm{ROI}}|\mathrm{G}(\mathrm{x}, \mathrm{y})|^{2}}
$$

where

$\left(\sum_{\mathrm{x}, \mathrm{y} \in \mathrm{ROI}}\left|\mathrm{G}(\mathrm{x}, \mathrm{y}) \| \mathrm{G}^{(\ell, \mathrm{m})}(\mathrm{x}, \mathrm{y})\right|\right) /\left(\sum_{\mathrm{x}, \mathrm{y} \in \mathrm{ROI}}\left|\mathrm{G}^{(\ell, \mathrm{m})}(\mathrm{x}, \mathrm{y})\right|^{2}\right)$

where $\mathrm{G}$ is the reconstruction of $\mathrm{H}$ and $\mathrm{G}^{(\ell, \mathrm{m})}$ is the reconstruction of $\mathrm{H}^{(\ell, \mathrm{m})}$. ROI denotes the spatial "region of interest";

2) the Diffraction Efficiency $\eta^{(\ell, m)}$ as given by

$$
\eta^{(\ell, \mathrm{m})}=\frac{\sum_{\mathrm{x}, \mathrm{y} \in \mathrm{ROI}}\left|\mathrm{G}^{(\ell, \mathrm{m})}(\mathrm{x}, \mathrm{y})\right|^{2}}{\mathrm{MN}}
$$

where $1 \leq \mathrm{x} \leq \mathrm{M}$ and $1 \leq \mathrm{y} \leq \mathrm{N}$.

The two criteria $\mathrm{E}^{(\ell, \mathrm{m})}$ and $\eta^{(\ell, \mathrm{m})}$ are antagonistic [20], [21] and the optimal-tradeoff between these two are obtained by minimizing a cost function, $\mathrm{C}^{(\ell, \mathrm{m})}$, formed using a linear combination of the two criteria [19]-[21]

$$
\mathrm{C}^{(\ell, \mathrm{m})}(\mu)=\mu \mathrm{E}^{(\ell, \mathrm{m})}+(1-\mu) / \eta^{(\ell, \mathrm{m})} \forall 0 \leq \mu \leq 1 .
$$

In (7), ' $\mu$ ' is a parameter which permits the weighting of the two criteria to be adjusted. To chose a desired tradeoff between the two criteria an Optimal Characteristic Curve (OCC) [20]-[22] is plotted. The OCC represents one criterion as a function of the other so that the cost function, in (6), is minimized. In our case, the Amplitude Error $\mathrm{E}^{(\ell, \mathrm{m})}$ is drawn as a function of the inverse diffraction efficiency $1 / \eta^{(\ell, \mathrm{m})}$ obtained for values of $\mathrm{H}^{(\ell, \mathrm{m})}$ that minimize (6) for different values of $0 \leq \mu \leq 1$. Thus the OCC permits us to choose the value of $\mu$ to achieve the desired tradeoff, which leads to the best set of values for the amplitude error and the diffraction efficiency.

If the complex conjugate of the wavefront retrieved at the CCD plane is displayed on the SLM, and illuminated by coherent light of the same wavelength as that used for recording, a real image is formed at the same distance from the SLM as the recording distance from the object to the CCD camera. This is true only if the CCD pixel size is the same as the SLM pixel size. The reconstruction distance of the hologram is different to the recording distance if either the pixel size of the SLM used for displaying the hologram is different to the CCD pixel size used to record the hologram, and/or the wavelength used for reconstruction is different to that used for recording. If the ratio of the pixel size of the SLM to that of the CCD is ' $\Omega$ ' (assuming square pixel in the CCD as well as in the SLM as is true in the present case), then the reconstruction distance ' $\mathrm{d}$ ' of a hologram recorded at a distance ' $\mathrm{z}$ ' is given by $d=\left(\lambda_{1}\right) /\left(\lambda_{2}\right) \Omega^{2} \mathrm{z}$, 
where $\lambda_{1}$ is the recording wavelength and $\lambda_{2}$ is the reconstruction wavelength (see Appendix II). Furthermore, there is a magnification by a factor ' $\Omega$ '. To achieve reconstruction at shorter distances, the SLM was placed at the front focal plane of a lens of focal length ' $f$ '. The reconstruction of the object wavefront is then obtained at a distance $d=\mathrm{f}\left(1+\left(\lambda_{2} \mathrm{f}\right) /\left(\Omega^{2} \lambda_{1} z\right)\right)$, with a magnification factor of $\mathrm{mf}=\left(\lambda_{2} \mathrm{f}\right) /\left(\Omega \lambda_{1} z\right)$ (see Appendix II). A quadratic output phase factor is introduced by the optical system, which is not significant if only the intensity of the reconstructed wavefront is of interest.

\section{EXPERIMENTS}

Our digital holograms are recorded using the optical setup (shown in Fig. 1) based on a Mach-Zehnder interferometer architecture in an in-line configuration. A spatially filtered linearly polarized helium-neon $(\lambda=632.8 \mathrm{~nm})$ laser beam is split into object and reference beams, both of which are spatially filtered and collimated. The first beam illuminates the $3 \mathrm{D}$ object placed at a distance $d$ from a 10-b 2032×2048 pixel CCD camera. The reference beam passes through either half-wave and/or quarter-wave plates. Through permutation of the fast and slow axes of the plates we can achieve phase shifts of $0, \pi / 2, \pi$, and $3 \pi / 2$ radians. The reference beam combines with the light diffracted from the object and forms an interference pattern in the plane of the camera. At each of the four phase shifts we record an interferogram. Using these four intensity images, the complex-valued camera-plane wavefront can be numerically extracted using phase-shift interferometric techniques [2]-[6].

To determine the Jones matrix of the SLM a Mach-Zehnder interferometer is used [23], [24]. The SLM is placed in one arm of a Mach-Zehnder interferometer. A split pattern with regions consisting of two gray levels - the gray level at which the Jones Matrix has to be estimated and a reference zero gray level - is displayed on the SLM [23], [24]. The parameters $\rho, \gamma$, and $\beta$ are estimated by measuring the shift in fringes formed due to interference of the light passing through the two regions displaying different gray levels (which are a function of the applied voltage ' $V$ '). A plot of the parameters $\rho, \gamma$, and $\beta$ characterized for the transmission SLM (Holoeye Model LC2002), for 15 sets of gray level values is shown in Fig. 2.

The in-line holograms of two objects: 1) 'PINS'-two pins located at distances of 288 and $316 \mathrm{~mm}$ away from the capturing CCD and 2) 'TOY' - A block toy at a distance $367 \mathrm{~mm}$ from the CCD were recorded using phase shifting interferometric technique. The wavelength used for recording the holograms was $633 \mathrm{~nm}$ and the CCD pixel size was $7.4 \mu \mathrm{m}$ with $2032 \times 2048$ pixels.

The amplitude and the phase of the complex valued wavefront retrieved at the recording plane is shown in Figs. 3(a)-(b) and 6(a)-(b). The histograms of the amplitude (normalized to 1) and phase values of the PINS are shown in Fig. 3(c)-(d) and that of TOY in Fig. 6(c)-(d). For the amplitude hologram the bin size was chosen as 0.1 and for the phase histogram the bin size was chosen as $\pi / 10$. For the TOY hologram $79 \%$ of the total values fall between 0 and 0.1 and $77 \%$ of the phase values fall between $-\pi / 10$ and $\pi / 10$. The corresponding values for PINS hologram are $81 \%$ and $76 \%$.

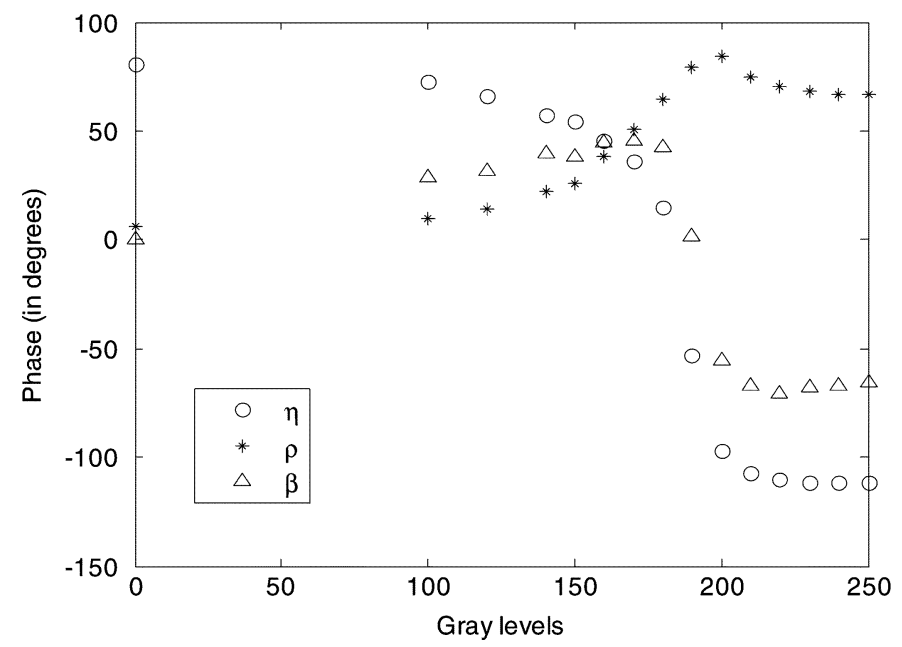

Fig. 2. Characterization of parameters $\rho, \gamma$, and $\beta$ of SLM (HOLOEYE LC2002). The contrast and brightness settings used were 255 and 100, respectively.

In each case, $512 \times 512$ center pixels of the complex valued hologram are mapped to the SLM modulation states. In performing the mapping we considered 48 distinct polarization states at the input and output of the SLM. The azimuth angles $\chi \in\left\{0^{\circ}, 30^{\circ}, 60^{\circ}, 90^{\circ}\right\}$ and retardance (angle) values $\varphi \in$ $\left\{-170^{\circ},-140^{\circ},-110^{\circ},-80^{\circ},-50^{\circ},-20^{\circ}, 10^{\circ}, 40^{\circ}, 70^{\circ}, 100^{\circ}\right.$, $\left.130^{\circ}, 160^{\circ}\right\}$ were examined.

The operating point was chosen to give both a low amplitude error as well as good diffraction efficiency $(0.016$ and 0.48 , respectively, for PINS and 0.26 and 0.44 , respectively, for TOY). The OCC for the hologram PINS is shown in Fig. 4. The input and output polarization states $P_{\text {in }}$ and $\mathrm{P}_{\text {out }}$ corresponding to the chosen operating point are $\chi_{\text {in }}=60^{\circ}, \varphi_{\text {in }}=70^{\circ}$ and $\chi_{\text {out }}=60^{\circ}, \varphi_{\text {out }}=-30^{\circ}$ for PINS and $\chi_{\text {in }}=60^{\circ}, \varphi_{\text {in }}=80^{\circ}$ and $\chi_{\text {out }}=60^{\circ}, \varphi_{\text {out }}=-30^{\circ}$ for TOY. As can be seen the optimal configuration of polarization elements are quite similar for the two holograms. This might be due to the fact that almost $80 \%$ of the values in both the holograms are the same.

The configuration of polarization elements, (linear polarizer and waveplate with retardance $\Gamma$ ), to realize these polarization states are calculated using the relations [18]

$$
\begin{aligned}
\sin 2\left(\theta_{L}-\theta_{W}\right) & =\frac{\sin \phi \sin 2 \psi}{\sin \Gamma} \\
\tan \theta_{W} & =-\frac{\cos \left(\frac{\phi-\Gamma}{2}\right)}{\cos \left(\frac{\phi+\Gamma}{2}\right)} \tan \left(\theta_{L}-\theta_{W}\right) .
\end{aligned}
$$

For a quarter waveplate $\left(\Gamma=90^{\circ}\right)$, the configuration of polarization elements at the input and output of SLM to generate and detect these polarization states are $\theta_{\mathrm{L}}=-44^{\circ}, \theta_{\mathrm{W}}=-71^{\circ}$ and $\theta_{\mathrm{L}}=-5^{\circ}, \theta_{\mathrm{W}}=8^{\circ}$ for PINS, and $\theta_{\mathrm{L}}=-52^{\circ}, \theta_{\mathrm{W}}=-81^{\circ}$ and $\theta_{\mathrm{L}}=-5^{\circ}, \theta_{\mathrm{W}}=8^{\circ}$. The complex values of the hologram were mapped to one of the SLM modulation states using the algorithm given in Appendix I.

The mapped holograms of dimension $512 \times 512$ pixels were displayed on the SLM (Holoeye Model LC2002, $832 \times 624$ pixels, pixel size $32 \mu \mathrm{m} \times 32 \mu \mathrm{m})$. The reconstruction of the 


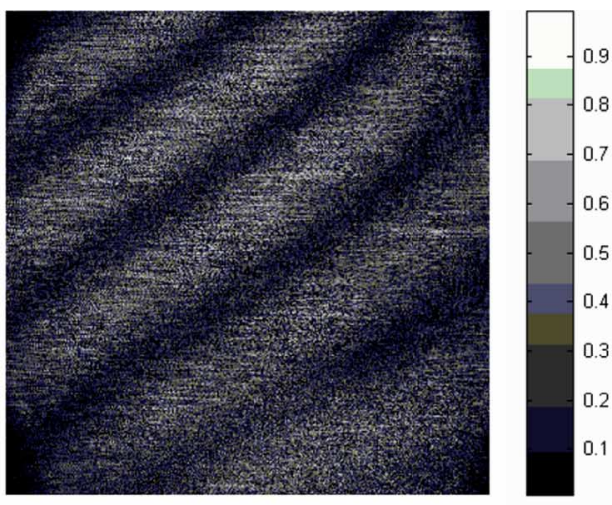

(a)

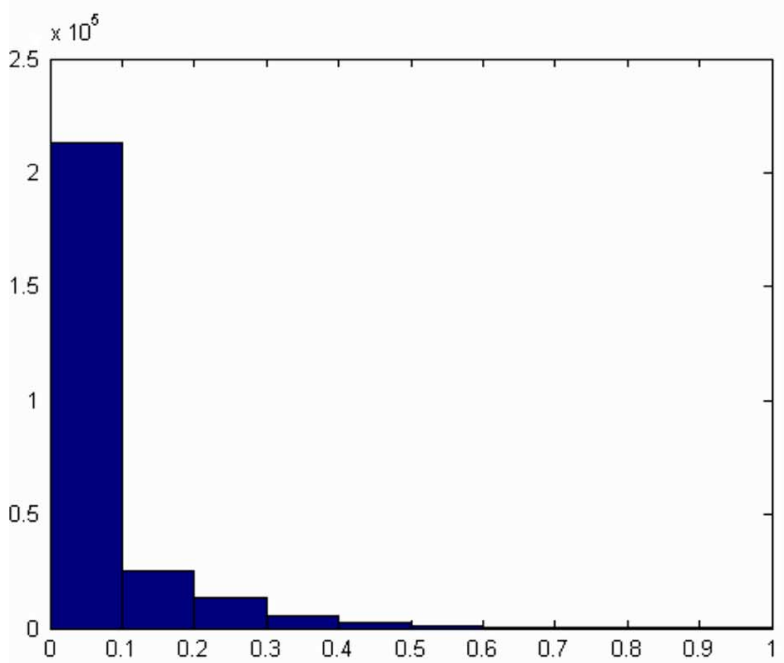

(c)

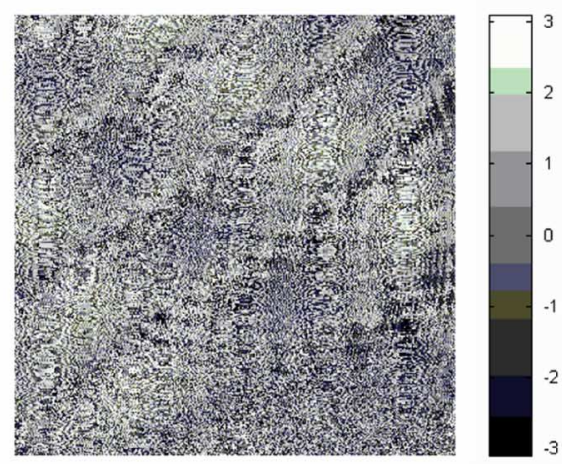

(b)

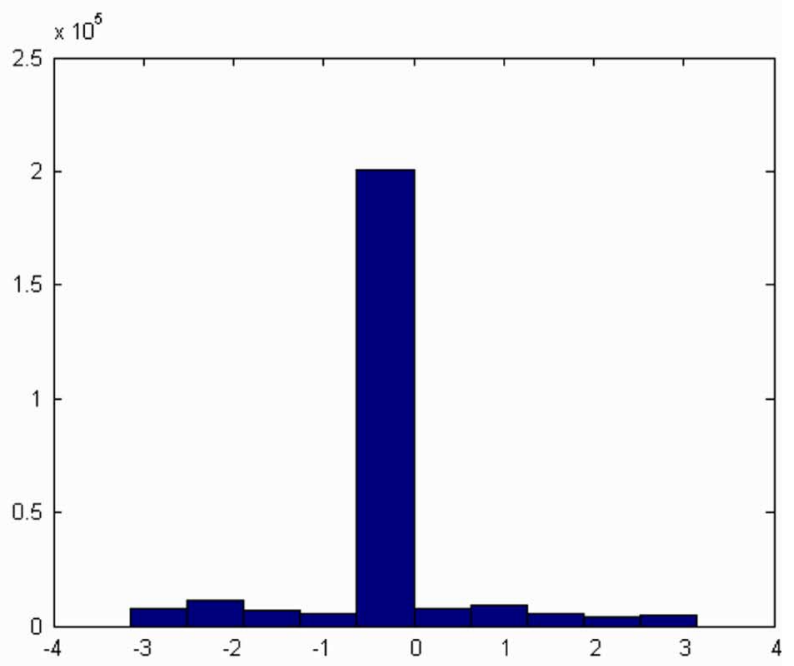

(d)

Fig. 3. (a) Amplitude and (b) phase of hologram PINS. (c) Histogram of amplitude and (d) phase of hologram PINS.

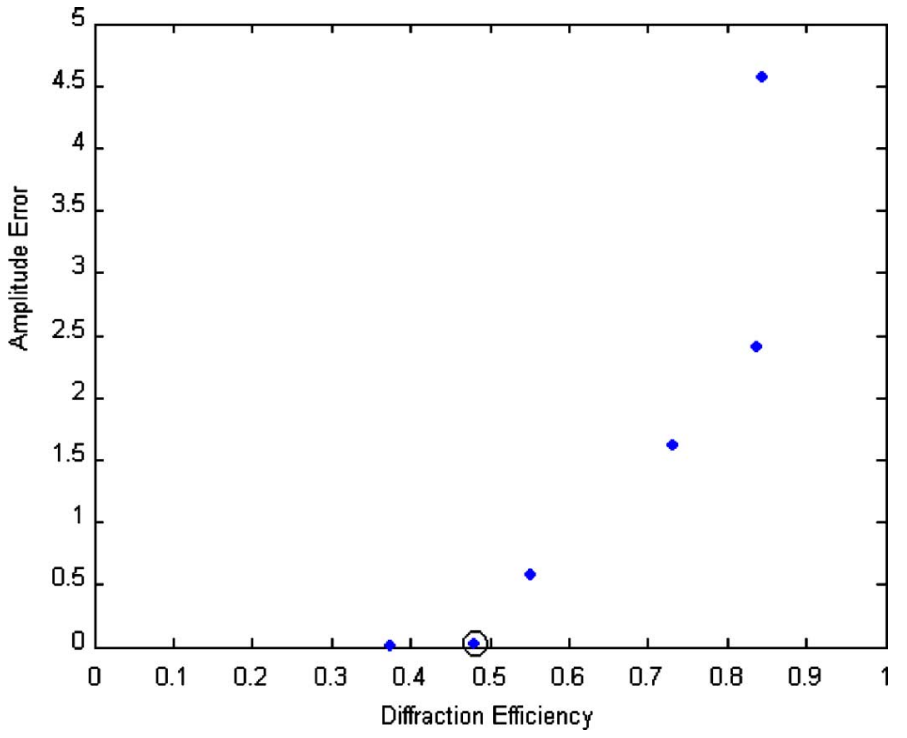

Fig. 4. OCC plot showing the tradeoff between Diffraction Efficiency and Amplitude Error as $\mu$ varies between 0 and 1 for the PINS hologram. The chosen vale of $\mu$ for the reconstructions in Fig. 5 is circled.

holograms displayed on the SLM was done using the wavelength $\lambda=532 \mathrm{~nm}$. The reconstruction distance is different to that used for recording the holograms as the pixel size of the SLM used for reconstruction is different (4.32 times bigger) to the pixel size of CCD used for recording the hologram. The reconstruction wavelength is alo different from the recording one. An analysis to account for the above two factors is given in Appendix II. Following the notation given in Appendix II, $\Omega=4.32$ and the reconstruction distance is thus $d=22.25 \mathrm{z}$. For the holograms recorded at distances $\mathrm{z}=288 \mathrm{~mm}$ and $316 \mathrm{~mm}$, the reconstruction distance would, therefore, be 6.4 and $7 \mathrm{~m}$, respectively and both are magnified by the factor of $\Omega=4.32$. The reconstruction distances can be brought closer by using a convex lens. The numerical reconstruction of the PINS hologram at distances $d=188$ and $216 \mathrm{~mm}$ are shown in Fig. 5(a)-(b). The corresponding numerical reconstructions using the holograms mapped onto the SLM is shown in Fig. 5(c)-(d). Using a lens of focallength $160 \mathrm{~mm}$, the reconstructions of the hologram PINS obtained at distances $d=16.68 \mathrm{~cm}$ and $d=16.77 \mathrm{~cm}$ from the lens, are shown in Fig. 5(e)-(f). Fig. 7(a)-(b) shows the numerical reconstructions of the hologram TOY and the hologram mapped to the SLM modulating states. Fig. 7(c) shows the encoded hologram which was displayed on the SLM and Fig. 7(d) shows the reconstructions at distances $d=19.5 \mathrm{~cm}$ from a lens of focal length $200 \mathrm{~mm}$. All the above numerical reconstructions were 


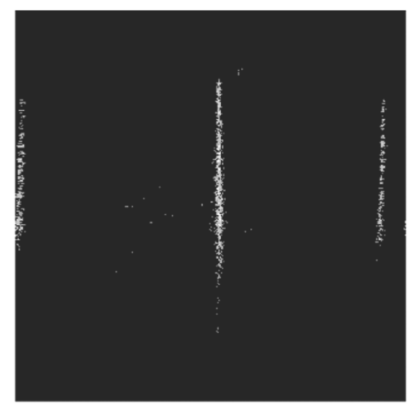

(a)

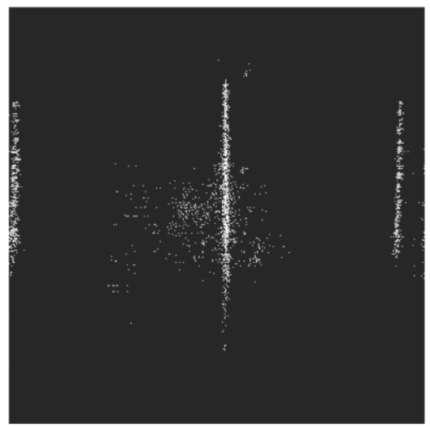

(c)

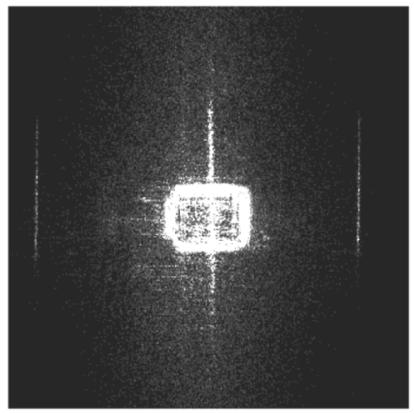

(e)

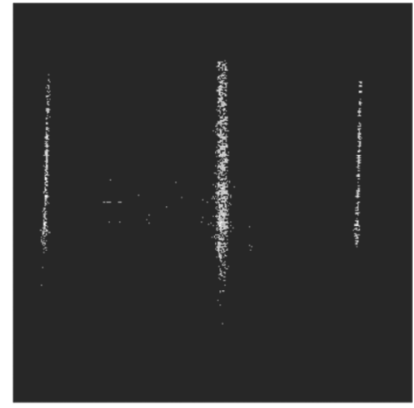

(b)

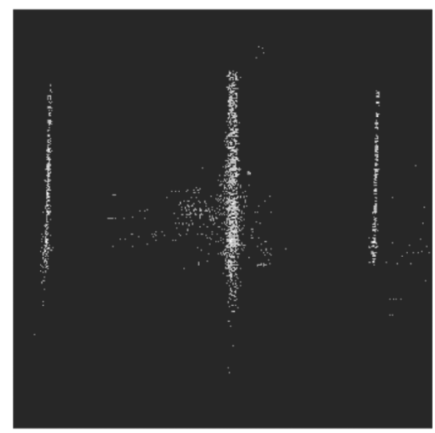

(d)

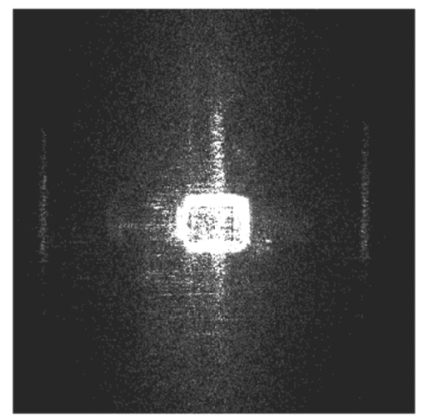

(f)
Fig. 5. (a) Numerical reconstruction of the hologram PINS at $d=188 \mathrm{~mm}$ and (b) $d=188 \mathrm{~mm}$. (c) Numerical reconstruction of the hologram displayed on SLM at $d=188 \mathrm{~mm}$ and (d) $d=188 \mathrm{~mm}$. (e) Optical reconstruction at $d=16.68 \mathrm{~cm}$, and (f) $d=16.77 \mathrm{~cm}$.

carried out using the $512 \times 512$ hologram pixels used for optical reconstructions. The numerical reconstructions does not take into account the optical system noises and the effects due to SLM fill factor.

From Figs. 5(c)-(d) and 7(b), a deterioration in quality is observed when the holograms encoded onto an SLM with 15 modulation states are numerically reconstructed as compared to those with fully complex-valued hologram. A further deterioration in quality is observed in the experimentally obtained optical reconstructions in Figs. 5(e)-(f) and 7(d) as compared to the numerical reconstructions. The difference in quality of the reconstructions of TOY hologram and PINS hologram is mainly due to the difference in the nature of objects used to record the holograms, PINS being more reflective than TOY.

In this paper, the metrics used to quantify the quality of reconstruction are amplitude error and diffraction efficiency. A low amplitude error and high diffraction efficiency is desired. The factors which affects the reconstruction quality can be attributed to the quality of holograms used for reconstruction and the SLM used. The factors that depend on SLM include the modulation states that constitute the chosen operating curve, the number of distinct modulation states available and the range of modulation states. The quality of holograms used also affects the reconstructions. A factor that affects the quality of hologram is the nature of objects used to record the holograms. This is reflected in the difference in quality of the reconstructions of TOY hologram and PINS hologram, mainly due to the difference in the nature of objects used to record the holograms, PINS being more reflective than TOY.

The holograms used in this paper were recorded with light reflected off the optically rough surfaces of real world objects resulting in speckles in the reconstruction. The speckles can be reduced using various digital post-processing techniques [11]. The holograms had some amount of residual conjugate term that also contributed to the deterioration in quality.

\section{CONCLUSION}

We discuss a projection system for real world 3D objects. The digital holograms of the 3D objects are recorded using an in-line phase shifting holographic setup. The complex object wavefront at the CCD camera plane, retrieved from the recorded holograms, is encoded to the modulation states of an SLM obtained by characterizing the Jones matrix associated with an SLM as a function of applied voltage. The modulation states of an SLM also depend on the configuration of polarization elements used in conjunction with the SLM. For a given set of holograms a method to find the mapped holograms as well as the configuration of polarization elements is described. We have presented some experimental results illustrating reconstruction and discussed some of the factors that can affect the optical reconstruction of the holograms. We analyze the case when the pixel sizes of the CCD and SLM used for recording the hologram and projection are different.

\section{APPENDIX \\ Algorithm to ENCODE Fully COMPLEX-VALUED SIGNAL ONTO AN SLM}

Step 1: Choose the input and output polarization states $P_{\ell}$ and $\mathrm{P}_{\mathrm{m}}$ from the discrete set, $\mathrm{P}_{\ell}, \mathrm{P}_{\mathrm{m}} \in$ $\left\{\mathrm{P}_{1}, \mathrm{P}_{2}, \ldots, \cdot \mathrm{P}_{\mathrm{k}}\right\}$.

Step 2: Calculate the SLM modulation states $\Gamma_{\mathrm{i}}^{(\ell, m)} \forall \mathrm{V}_{\mathrm{i}} \in$ $\left\{\mathrm{V}_{1}, \ldots, \mathrm{V}_{\mathrm{n}}\right\}$ for the chosen polarization states.

Step 3: Calculate $\left|\mathrm{H}(\mathrm{x}, \mathrm{y}) \Gamma^{(\ell, \mathrm{m})}\right| \forall \Gamma^{(\ell, \mathrm{m})} \in$ $\left\{\Gamma_{1}^{(\ell, \mathrm{m})}, \Gamma_{2}^{(\ell, \mathrm{m})}, \ldots, \Gamma_{\mathrm{n}}^{(\ell, \mathrm{m})}\right\}$, where $\mathrm{H}(\mathrm{x}, \mathrm{y})$ is the $(x, y)$ th pixel of the hologram. Assign $\mathrm{H}^{(\ell, \mathrm{m})}(\mathrm{x}, \mathrm{y})=\Gamma_{\mathrm{i}}^{(\ell, \mathrm{m})}$, where $\Gamma_{\mathrm{i}}^{(\ell, \mathrm{m})}$ is the value of $\Gamma^{(\ell, \mathrm{m})}$ which minimizes $\left|\mathrm{H}(\mathrm{x}, \mathrm{y}) \Gamma^{(\ell, \mathrm{m})}\right| . \mathrm{H}^{(\ell, \mathrm{m})}$ is the estimated hologram corresponding to the polarization states $\mathrm{P}_{\ell}$ and $\mathrm{P}_{\mathrm{m}}$. Repeat Step 3 for all the hologram pixels.

Step 4: Obtain the reconstruction $\mathrm{G}^{(\ell, \mathrm{m})}$ of the estimated hologram $\mathrm{H}^{(\ell, \mathrm{m})}$.

Step 5: Calculate the Amplitude Error, $\mathrm{E}^{(\ell, \mathrm{m})}$, and the Diffraction Efficiency, $\eta^{(\ell, \mathrm{m})}$, using (4) and (5). 


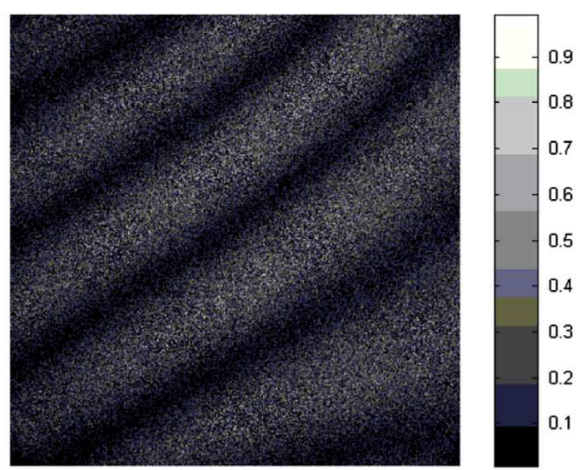

(a)

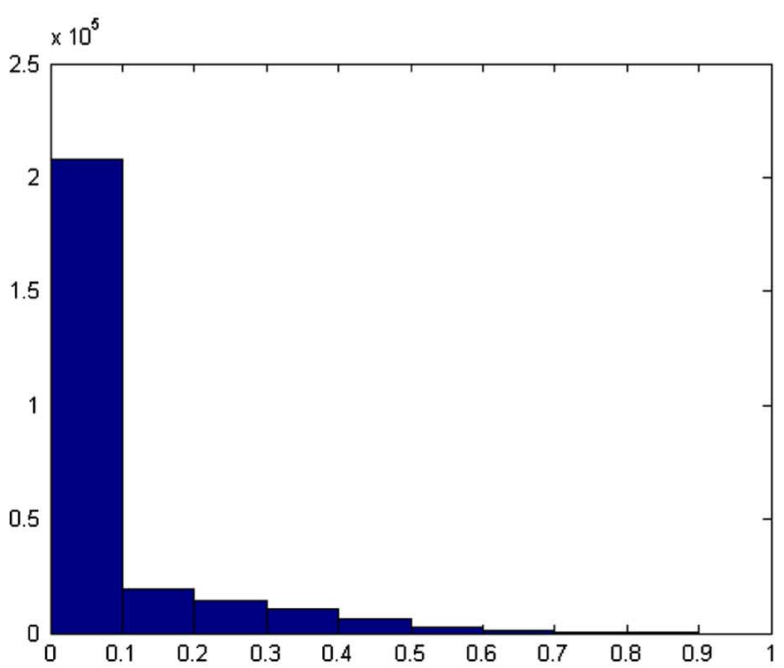

(c)

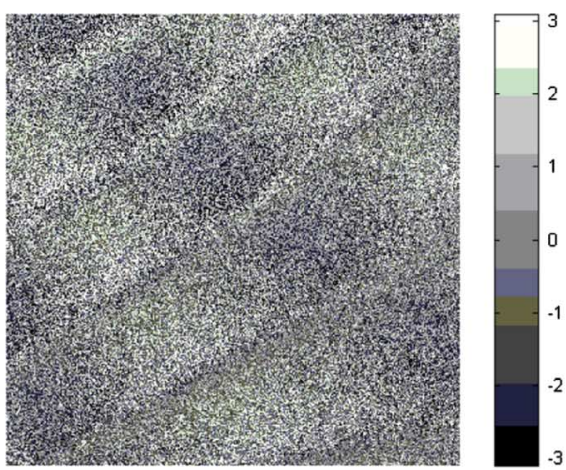

(b)

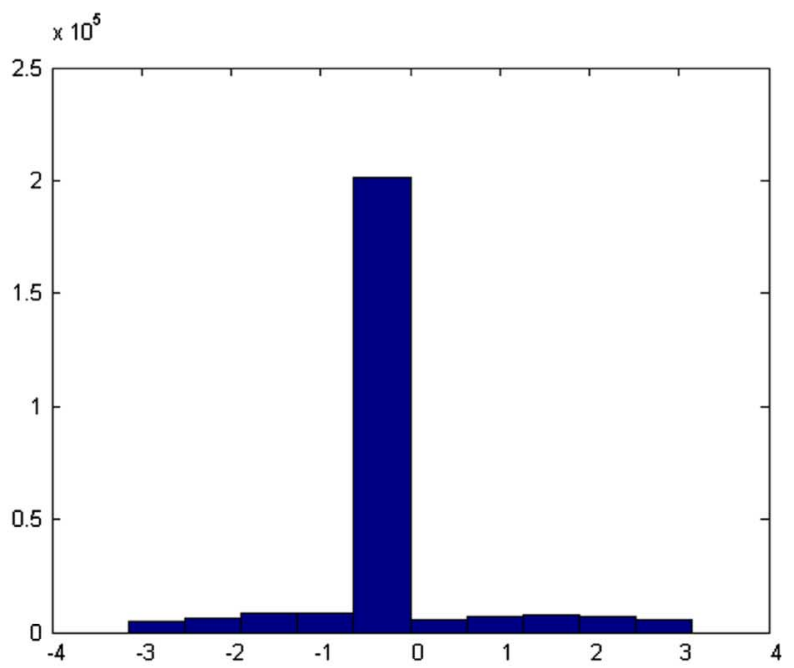

(d)

Fig. 6. (a) Amplitude and (b) phase of hologram TOY. (c) Histogram of the amplitude and (d) phase of hologram TOY.

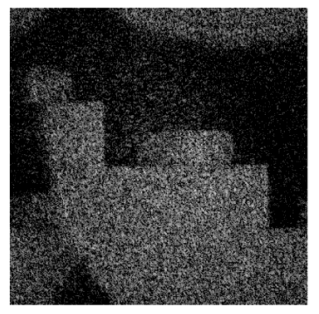

(a)

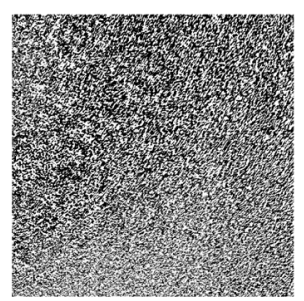

(c)

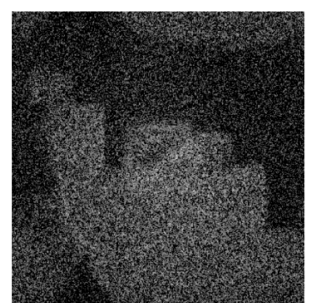

(b)

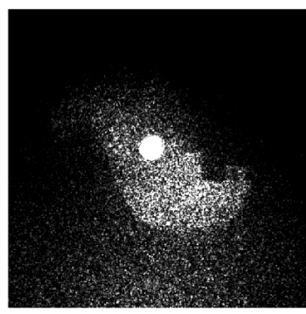

(d)
Fig. 7. (a) Numerical reconstruction of the hologram TOY at $d=367 \mathrm{~mm}$. (b) Numerical reconstruction of the hologram displayed on SLM at $d=367$ mm. (c) Encoded image displayed on SLM. (d) Optical reconstruction at 19.5 $\mathrm{cm}$ from a lens of focal length $20 \mathrm{~cm}$.

Repeat Steps 1 to 5 for all the input-output polarization states $\left(\mathrm{P}_{\ell}, \mathrm{P}_{\mathrm{m}}\right) \in$ $\left\{\left(\mathrm{P}_{1}, \mathrm{P}_{1}\right),\left(\mathrm{P}_{1}, \mathrm{P}_{2}\right), \ldots,\left(\mathrm{P}_{\mathrm{K}}, \mathrm{P}_{1}\right), \ldots,\left(\mathrm{P}_{\mathrm{k}}, \mathrm{P}_{\mathrm{k}}\right)\right\}$.
Step 6: Calculate the cost function $\mathrm{C}^{(\ell, \mathrm{m})}(\mu)$ from (6), for $\mu$ ranging from 0 to 1 for all the input-output polarization states $\left(\mathrm{P}_{\ell}, \mathrm{P}_{\mathrm{m}}\right) \in$ $\left\{\left(\mathrm{P}_{1}, \mathrm{P}_{1}\right),\left(\mathrm{P}_{1}, \mathrm{P}_{2}\right), \ldots,\left(\mathrm{P}_{\mathrm{K}}, \mathrm{P}_{1}\right), \ldots, \cdot\left(\mathrm{P}_{\mathrm{k}}, \mathrm{P}_{\mathrm{k}}\right)\right\}$.

Step 7: Find $\operatorname{Min}\left\{\mathrm{C}^{(\ell, \mathrm{m})}(\mu)\right\}$ for a given value of $\mu$. Let $\mathrm{E}^{(\ell, \mathrm{m})}(\mu)$ and $\eta^{(\ell, \mathrm{m})}(\mu)$ be the values corresponding to $\operatorname{Min}\left\{\mathrm{C}^{(\ell, \mathrm{m})}(\mu)\right\}$. Plot the OCC of $\mathrm{E}^{(\ell, \mathrm{m})}(\mu)$ as a function of $\eta^{(\ell, \mathrm{m})}(\mu)$ for $\mu$ ranging from 0 to 1 . Choose the operating point in the OCC $\left[\mathrm{E}^{(\ell, \mathrm{m})}(\mu *), \eta^{(\ell, \mathrm{m})}(\mu *)\right]$ for $\mu=\mu *$, the value that achieves the desired trade-off, i.e., $\mathrm{E}^{(\ell, \mathrm{m})}(\mu *)$ is sufficiently low and $\eta^{(\ell, \mathrm{m})}(\mu *)$ is sufficiently high. The input and output polarization states $\mathrm{P}_{\ell}$ and $\mathrm{P}_{\mathrm{m}}$ corresponding to the chosen operating point determines the configuration of polarization elements at the input and output to the SLM.

\section{APPENDIX}

Consider a digital holographic recording setup as shown in Fig. 1. Let the distance between the object and the CCD recording plane be ' $z$ '. Let the recording wavelength be ' $\lambda_{1}$ '. Consider the case in which the reconstruction is performed by propagating the complex conjugate of the object wavefront by a distance ' $d$ '. Let ' $\lambda_{2}$ ' be the reconstruction wavelength, and ' $\Omega$ ' the ratio of the SLM pixel size to CCD pixel size. Using the 
$\mathrm{ABCD}$ formalism [25], the wavefront from the object plane to reconstruction plane is seen to have undergone a transformation in the phase space as given by

$$
\left[\begin{array}{l}
\mathrm{x}_{2} \\
\mathrm{k}_{2}
\end{array}\right]=\left[\begin{array}{lll} 
& & \mathrm{B} \\
\mathrm{C} & \mathrm{D} &
\end{array}\right]\left[\begin{array}{l}
\mathrm{x}_{1} \\
\mathrm{k}_{1}
\end{array}\right]
$$

where

$$
\begin{aligned}
{\left[\begin{array}{ll}
\mathrm{A} & \mathrm{B} \\
\mathrm{C} & \mathrm{D}
\end{array}\right] } & =\left[\begin{array}{cc}
1 & \lambda_{2} d \\
0 & 1
\end{array}\right]\left[\begin{array}{cc}
\Omega & 0 \\
0 & \frac{1}{\Omega}
\end{array}\right]\left[\begin{array}{cc}
1 & -\lambda_{1} \mathrm{z} \\
0 & 1
\end{array}\right] \\
& =\left[\begin{array}{cc}
\Omega & -\lambda_{1} \mathrm{Z} \Omega+\frac{\lambda_{2} d}{\Omega} \\
0 & \frac{1}{\Omega}
\end{array}\right] .
\end{aligned}
$$

Setting $B=0$, for reconstruction plane to correspond to imaging geometry we have,

$$
d=\frac{\lambda_{1} \mathrm{z} \Omega^{2}}{\lambda_{2}}, \quad \text { and magnification } \mathrm{M}=\Omega .
$$

For the case, where the reconstruction geometry is as shown in Fig. 1, where the SLM is at the front focal plane of the lens of focal length ' $f$ ' and the reconstruction plane is at a distance ' $d$ ' behind the lens, it can be shown that

$$
\begin{aligned}
{\left[\begin{array}{ll}
\mathrm{A} & \mathrm{B} \\
\mathrm{C} & \mathrm{D}
\end{array}\right]=\left[\begin{array}{cc}
1 & \lambda_{2} d \\
0 & 1
\end{array}\right]\left[\begin{array}{cc}
1 & 0 \\
-1 / \lambda_{2} \mathrm{f} & 1
\end{array}\right] } \\
\times\left[\begin{array}{cc}
1 & \lambda_{2} \mathrm{f} \\
0 & 1
\end{array}\right]\left[\begin{array}{cc}
\Omega & 0 \\
0 & \frac{1}{\Omega}
\end{array}\right]\left[\begin{array}{cc}
1 & -\lambda_{1} \mathrm{z} \\
0 & 1
\end{array}\right] .
\end{aligned}
$$

Thus $\mathrm{A}=\Omega(1-(d / \mathrm{f})) ; \mathrm{B}=-\lambda_{1} \mathrm{z} \Omega+\left(\lambda_{2} \mathrm{f}\right) /(\Omega)+$ $\left(\lambda_{1} \mathrm{dz} \Omega\right) /(\mathrm{f}) ; \mathrm{C}=(-\Omega) /\left(\lambda_{2} \mathrm{f}\right) ;$ and $\mathrm{D}=\left(\lambda_{1} z \Omega\right) /\left(\lambda_{2} \mathrm{f}\right)$.

For the reconstruction plane to correspond to an imaging plane, $\mathrm{B}=0$

$$
d=\mathrm{f}\left(1-\frac{\lambda_{2} \mathrm{f}}{\lambda_{1} z \Omega^{2}}\right) \quad \text { and } \quad \mathrm{M}=\Omega\left(1-\frac{d}{\mathrm{f}}\right) .
$$

\section{ACKNOWLEDGMENT}

The authors thank the reviewers for their comments.

\section{REFERENCES}

[1] U. Schnars and W. P. O. Jüptner, "Digital recording and numerical reconstruction of holograms," Meas. Sci. Technol., vol. 13, 2002, R85R101.

[2] Y. Frauel, T. J. Naughton, O. Matoba, E. Tajahuerce, and B. Javidi, "Three-dimensional imaging and processing using computational holographic imaging," Proc. IEEE, vol. 94, pp. 636-653, ??? 2006.

[3] J. Maycock, C. P. Mc Elhinney, B. M. Hennelly, T. J. Naughton, J. B. Mc Donald, and B. Javidi, "Three-dimensional scene reconstruction of partially occluded objects using digital holograms," Appl. Opt., vol. 45, pp. 2975-2985, 2006

[4] T. J. Naughton, J. B. Mc Donald, and B. Javidi, "Efficient compression of digital holograms for Internet transmission of three-dimensional images," Appl. Opt., vol. 42, pp. 4758-4764, 2003.

[5] T. J. Naughton, Y. Frauel, B. Javidi, and E. Tajahuerce, "Compression of digital holograms for three-dimensional object reconstruction and recognition," Appl. Opt., vol. 41, pp. 4124-4132, 2002.

[6] B. Munjuluri, M. L. Huebschman, and H. R. Garner, "Rapid hologram updates for real-time volumetric information displays," Appl. Opt., vol. 44, pp. 5076-5085, 2005.
[7] T. Ito and T. Shimobaba, "One-unit system for electro-holography by use of a special purpose computation chip with a high-resolution liquidcrystal display toward a three dimension television," Opt. Exp., vol. 12, pp. 1788-1793, 2004.

[8] M. L. Huebschman, B. Munjuluri, and H. R. Garner, "Dynamic holographic 3-D image projection," Opt. Exp., vol. 11, pp. 437-445, 2003.

[9] T. Haist, M. Schonleber, and H. J. Tiziani, "Computer-generated holograms from 3D-objects written on twisted-nematic liquid crystal displays," Opt. Commun., vol. 140, pp. 299-308, 1997.

[10] M. Sutkowski and M. Kujawinska, "Application of liquid crystal (LC) devices for optoelectronic reconstruction of digitally stored holograms," Opt. \& Lasers in Eng., vol. 33, pp. 191-201, 2000.

[11] O. Matoba, T. J. Naughton, Y. Frauel, N. Bertaux, and B. Javidi, "Real-time three-dimensional object reconstruction by use of a phase-encoded digital hologram," Appl. Opt., vol. 41, pp. 6187-6192, 2002.

[12] O. Matoba, K. Hosoi, K. Nitta, and T. Yoshimura, "Fast acquisition system for digital holograms and image processing for three-dimensional display with data manipulation," Appl. Opt., vol. 45, pp. 8945-8950, 2006.

[13] M. W. Farn and J. W. Goodman, "Optimal maximum correlation filter for arbitrarily constrained devices," Appl. Opt., vol. 28, pp. 3362-3366, 1989.

[14] R. D. Juday, "Optimal realizable filters and the minimum Euclidean distance principle," Appl. Opt., vol. 32, pp. 5100-5111, 1993.

[15] R. D. Juday, "Correlation with spatial light modulator having phase and amplitude cross coupling," JOSA A, vol. 28, pp. 4865-4868, 1989.

[16] P. Birch, R. Young, D. Budgett, and C. Chatwin, "Dynamic complex wave-front modulation with an analog spatial light modulator," Opt. Lett., vol. 26, pp. 920-922, 2001.

[17] R. W. Cohn, "Pseudorandom encoding of complex-valued functions onto amplitude-coupled phase modulators," JOSA A, vol. 15, pp. 868-883, 1998.

[18] J. Nicolas, J. Campos, and M. J. Yzuel, "Phase and amplitude modulation of elliptical polarization states by non-absorbing anisotropic elements: Application to liquid crystal devices," J. Opt. Soc. Amer. A, vol. 19, pp. 1013-1020, 2002.

[19] C. R. Fernandez-Pousa, I. Moreno, N. Bennis, and C. Gomez-Reino, "Generalized formulation and symmetric properties of reciprocal nonabsorbing polarization devices: Application to liquid crystal display," J. Opt. Soc. Amer. A, vol. 17, pp. 2074-2080, 2000.

[20] C. Stolz, L. Bigue, and P. Ambs, "Implementation of high resolution diffractive optical elements on coupled phase and amplitude spatial light modulator," Appl. Opt., vol. 40, pp. 6415-6424, 2001.

[21] L. Legeard, P. Refregier, and P. Ambs, "Multicriteria optimality for iterative encoding of computer-generated holograms," Appl. Opt., vol. 36, pp. 7444-7449, 1997.

[22] V. Laude and P. Refregier, "Filter design for optical pattern recognition: Multicriteria optimization approach," Opt. Lett., vol. 15, pp. 84-856, 1990.

[23] U. Gopinathan, T. J. Naughton, and J. T. Sheridan, "Polarization encoding and multiplexing of two-dimensional signals: Application to image encryption," Appl. Opt., vol. 45, pp. 5693-5700, 2006.

[24] U. Gopinathan, D. S. Monaghan, T. J. Naughton, and J. T. Sheridan, "Polarization encoding multiplexing and wavefront modulation using Spatial light modulators: Application to image encryption and holographic displays," presented at the Summer School on Adaptive Optics and Microoptics, Humboldt-University of Berlin, Germany, Aug. 7-11, 2006 [Online]. Available: http://www.optecbb.de/summerschool/lectures/31\%20-\%20Fri\%2011.00\%20-\%20Unnikrishnan\%20Gopinathan.pdf, Invited talk, unpublished

[25] B. M. Hennelly and J. T. Sheridan, "Generalising optimizing and inventing numerical algorithms for the fractional Fourier, Fresnel and linear canonical transforms," JOSA A, vol. 22, pp. 917-927, 2005.

Unnikrishnan Gopinathan received the Ph.D. degree from Indian Institute of Technology, New Delhi, India.

He is currently a Humboldt research fellow at Stuttgart University, Stuttgart, Germany. His research interests include optical signal processing related to holographic displays and biomedical imaging. 


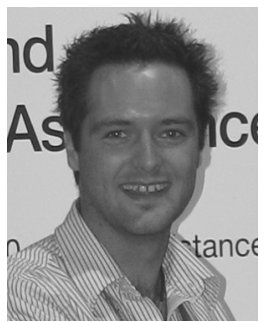

David S. Monaghan was born in 1982 in Ireland. $\mathrm{He}$ received the B.E. (hons.) degree in electronic engineering from University College Dublin (UCD) in 2004. This led him to pursue further studies within the Department of Electronic Engineering and he is currently working toward the Ph.D. degree in applied optics and optical encryption.

He has been actively involved in the UCD SPIE student chapter for the past three years. His research interests include optical encryption, optical signal processing, digital holography and spatial light modulator application with a view to optical encryption/decryption. His current research involves analyzing the Double Random Phase encoding algorithm and modelling of the physical behavior of spatial light modulators in paraxial optical systems.

Bryan M. Hennelly received the B.E. degree in electronic engineering from University College Dublin in 2001, and the Ph.D. degree in optical engineering in 2004.

He has worked as a temporary lecturer in the Department of Electrical and Electronic Engineering from 2004-2005 teaching optics, digital electronics and electronic engineering to final year students. His Ph.D. consisted of three separate theses on optical encryption, the discrete modelling of optical systems and metrology. After a year of lecturing he took a position as a post-doctoral researcher in the optics group in the department of Computer Science, National University of Ireland Maynooth (NUIM). His main focus of research is now digital holographic microscopy and also discrete signal processing. He is also an active researcher in the area of phase-space optics and has recently been invited by McGraw-Hill to co-edit a book on the subject and by the online journal Advances in Optical Technologies to be a guest editor on the same subject. He is currently a fellow of the Irish Research Council for Science, Engineering and Technology under the Embark Initiative Scheme. He is to be the principal investigator for NUIM in a recently awarded EC FP7 grant in digital holography and has published over 60 journal and conference proceeding papers.

Conor P. Mc Elhinney studied computer science and software engineering at National University of Ireland Maynooth (NUIM) and graduated with a bachelors degree in 2004. He then joined the computer vision and imaging laboratory at NUIM working in the holographic image processing group. He is now working toward the Ph.D. degree in the field of digital holographic image processing.

Damien P. Kelly received the Ph.D. degree from the School of Electrical, Electronic \& Mechanical Engineering, College of Engineering, Mathematical \& Physical Sciences, University College Dublin, Ireland, in 2007. He received the Bachelors degree in electronic engineering in University College Cork in 2000.

$\mathrm{He}$ is currently in Technical University of Vienna. His research interests include optical signal processing, terahertz spectroscopy systems, numerical and analytical modeling of optical systems, and speckle and statistical optics.

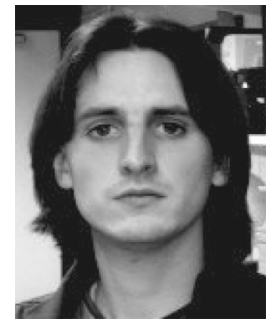

John B. McDonald (M'05) received the B.Sc. degree in 1996 and is currently working towards the Ph.D. degree in computer vision both at the National University of Ireland (NUI), Maynooth.

$\mathrm{He}$ has been a lecturer at the Department of Computer Science at NUI Maynooth since 1998. In 2002 he co-founded the Computer Vision and Imaging Laboratory which focuses on imaging science, computer vision and digital holography. His research interests include computer vision and pattern recognition, facial image processing and analysis, multiple view vision systems, intelligent vehicle systems, and digital holography. He was Chair of the International Machine Vision and Image Processing Conference 2007. He has published over 45 papers in archival journals and refereed conferences proceedings and holds two patents.

Mr. McDonald is a member of the IAPR.

Thomas J. Naughton received the B.Sc. degree (double hons.) in computer science and experimental physics from the National University of Ireland (formerly St. Patrick's College), Maynooth, in 1995.

He has worked at Space Technology, Ltd., Ireland, and has been a Visiting Researcher at the Department of Radioelectronics, Czech Technical University, Prague, Czech Republic, and the Department of Electrical and Computer Engineering, University of Connecticut, Storrs. He is currently a Lecturer in the Department of Computer Science, National University of Ireland, Maynooth, where he leads research groups in optical information processing, computer theory (optical and biological models of computation), distributed computing, and bioinformatics. He has published over 20 international journal articles and book chapters in these areas.

John T. Sheridan received the B.E.(H1) degree in electronic engineering, from University College Galway, (NUI), in 1985, the M.Sc.E.E. degree from Georgia Tech, Atlanta, in 1986, and the Ph.D. degree from Oxford University, Oxford, U.K., in 1991.

This was followed by postdoctoral fellowships, supported first (1991) by the Alexander von Humboldt Foundation and later (1992) by a European Community Bursary (Human Capital and Mobility), within the Physikalisches Institut of Friedrich-Alexander-Universitaet Erlangen-Nurnberg at the Lehrstuhl fur Angewandte Optik. In 1994 he took up a position as a visiting scientist at the European Commission Joint Research Centre in Italy. In 1997 he was appointed a Permanent Lecturer within the School of Physics, Dublin Institute of Technology. He joined the Dept of Electronic \& Electrical Engineering, University College Dublin in 2000, and is currently Deputy Director of the Optoelectronic Research Centre. He has authored nearly 120 reviewed journal papers and nearly 100 conference proceedings papers. 